\title{
A Review on Genetic Algorithm for Fault Tolerance in Distributed Sensor Networks
}

\author{
Prof. R. K. Krishna ${ }^{1}$, Anagha Nanoti ${ }^{2}$ \\ Department of Electronics, Rajiv Gandhi College of Engineering, Research \& Technology, Chandrapur ${ }^{1}$ \\ Department of Computer Technology, Rajiv Gandhi College of Engineering, Research \& Technology, Chandrapur ${ }^{2}$
}

\begin{abstract}
A considerable amount of attention and research has been devoted in recent years to the deployment of Distributed Sensor Networks (DSN) for use in data gathering, healthcare, collaborative information processing and environmental monitoring. This is the Survey paper for study of various Genetic Algorithms to be applied on Distributed Sensor Networks for better performance and results. However these networks are vulnerable to failure eventually due to many reasons. Hence the best suitable GA is to be applied. The main objective of proposed work is to organize such a network which consumes less energy and fault tolerant using Genetic Algorithms. Thus primary stage is to analyze requirements and preliminaries and accordingly plan for solving fault tolerance in Distributed Sensor Networks.
\end{abstract}

Keywords: DSN, Deployment, Sensor nodes, Genetic Algorithm, Population.

\section{INTRODUCTION}

A DSN is a high level distributed set of sensors that are interconnected by a communication network in the environment. The major requirement used in DSNs is scalability, security, production cost etc. Some of the unique characteristics of DSN are application specific, scale, density and deployment. The sensors are deeply embedded devices that are integrated with a physical environment and capable of acquiring signals, processing the signals, communicating, context aware computing and performing simple computation tasks. While this new class of networks has the potential to enable wide range of applications, it also pose serious challenges like routing, data gathering and dissemination, frequent network topology change, and fault Tolerance. There are various levels of faults in DSN environment such as: at node level or sink node level and network level faults (link failure and packet error). In this work, it has been considered that a node level fault and replace those nodes in the network.

The Distributed sensor Networks (DSN) framed with Genetic Algorithm. The Genetic Algorithm is metaheuristic inspired algorithm belonging to large class of evolutionary algorithm. The father of GA was John Holland invented in 1970. He represented intelligent exploitation of random search. GAs are used to generate high quality solution to optimization and search problems. Genetic Algorithms were invented to mimic some of processes observed in natural evolution. GA simulates "survival of fittest" among individuals over consecutive generation for solving problems. This principle was given by Charles Darwin. He stated that fittest individual dominates over weaker ones. Individuals in population are made to go through a process of evolution. Each individual is coded as finite length vector of components or variables, in terms of some alphabet, usually $\{0,1\}$. GA aims to use selective breeding of solutions to produce offsprings better than parents by combining information from chromosomes. Thus GAs will find better solutions in reasonable amount of time and applied for bigger problems (NP Hard problems) such as optimization.

\section{LITERATURE REVIEW}

Lokesh B. Bhajantri, N. Nalini, S. R. Nagaraj, H. V. Chaitra and N Srinivas[3] have proposed Routing, power management and data dissemination protocols have been specifically designed for DSN, where energy consumption is an essential design issues for routing. Maximizing the lifetime of the sensor nodes, it is preferable to distribute the energy dissipated throughout the sensor network in order to minimize the maintenance and maximize overall system performance. Optimization of routing method is an essential for routing in DSN because long communication distances between distributed sensor nodes and sink node in a network can greatly drain the energy of sensors and decrease the lifetime of the network.

N. Thangadurai, Dr. R. Dhanasekaran, and R. Pradeep [4] have given paper which mainly focuses on various models and different types of crossover implementations of existing GAs and we proposed a new GA model to minimize total energy consumption and provide better energy efficiency compare to other existing GAs techniques to extend the lifetime of networks.

M. Senthil, K. Sugashini, M. Abirami, N. Vaigai have given [5] work in which the goal is achieved by framing WSN with distributed hash table (DHT) which provides an efficient recovery method known as Checkpoint recovery and the topology is constructed using this table in a decentralized approach. The randomly directed 
Vol. 5, Issue 12, December 2016

exploration (RDE) of this approach uses the Adhoc on or divide it into multiple isolated sub-populations. Demand distance Vector (AODV) routing protocol to find Basically three types of GAs are present, they are-

the repaired node directly. The proposed method finds the repaired node directly and recovers malfunctioning node, consumption of time and energy is reduced thereby increases the performance level.

Ravneet Kaur, Neeraj Sharma. They gave-Dynamic Node Recovery for Improved Throughput in MANET. This paper compares the proposed dynamic node recovery technique with an existing base approach. The proposed research work ensures successful retrieval of checkpoints in case of node failures, thereby reducing the recovery time considerably. As an outcome, this improves throughput of the system. Trust factor of a cluster, in the proposed work, has significant impact on the count variable maintained by each node. The two are inversely proportional to each other. Count variable is kept track of so as to carry out the checkpointing task. When the count exceeds pre-specified threshold value, checkpointing is done. Prof. Prashant P. Rewagad, Satish R. Suryawanshi have discussed that Identity deceptions attacks are hard to detect attack [7]. e.g. sinkhole attack, wormhole attack, Sybil attacks. Trust aware routing framework based on trust values of sensor nodes is proved effective for preventing Identity deception attacks. But DOS attacks or link failure conditions are not discussed in existing system. This paper focuses on the study behavior of Trust aware routing framework with backup path generation algorithm for link failure recovery. Link failure happens during packet routing results into packet loss. We have to recover that link failure and avoid packet loss and increasing packet delivery by using recovery phenomena of backup path generation for making routing framework robust.

S.Sathish, A. Lawrance Ramesh, G. Sarath Kumar are the authors. They have contributed work on Node Recovery from a Failure in Wireless Sensor Networks [8]. This survey analyse the node recovery from a failure in Wireless Sensor Networks. In wireless sensor network, node recovery and node restoration is an active area for research. In this paper, we classify the node recovery process into two broad categories: (i) Recovery by node reposition and (ii) Replace by relay node placement. There are many schemes has been published based on these two approaches. This paper also analyzes the node recovery with consideration of topology changes. In this survey, paper we highlighting their strengths and limitation of each technique. This survey provides valuable ideas about node recovery in wireless sensor networks

\section{A KIND OF SURVEY}

A Survey on different types of Genetic Algorithms to be used which will be best suited is done. Genetic algorithms (GAs) are powerful search techniques that are used successfully to solve problems in different aspects. GAs is easy to implement and provide substantial gains in performance. Divide and conquer approach can be applied to GAs in different ways. One can divide GAs according to parallelization methods such as- Use a single population

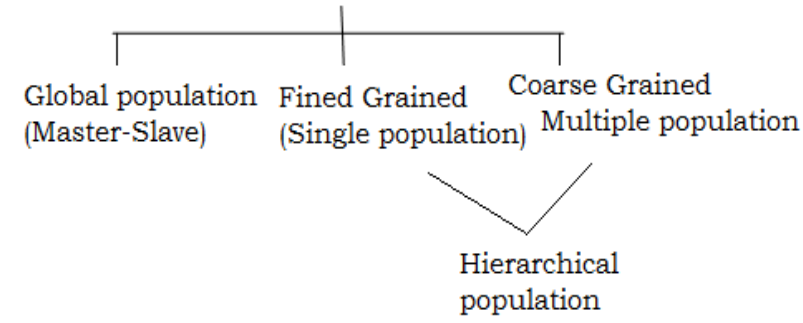

1) Master-Slave:

It uses single parametric population but evaluation of fitness is distributed among multiple processors.

Selection and crossover considers entire population known as Global Parallel GAs. Master-Slave Method does not affects the beheviour of algorithm. It goes with random mating of any two individuals in population.

\section{2) Fined Grained:}

Suited for massively parallel computers and consist of spatially structured population. Selection and mating are restricted to neighbor-hoods but overlap permits some interactions among all individuals. Ideal case is to have one individual for one processing element.

3) Coarsed Grained:

Consist of several Subpopulations which exchange individuals occasionally.

They are also called as distributed GAs, because they are implemented on distributed memory MIMD systems.

They are also known as Island GAs and are popularly used. One can further develop hybrid model from these three types for solving different problems depending on their complexity. Fined Grained and Coarsed Grained types of GAs can be combined to form Hierarchical GA.

4) Hierarchical GAs:

To parallelize GAs this method combines Multiple deme (Coarsed Grained) with Master-Slave GAs or Fined Grained. It has some spatially distributed population combines benefit of its components.

Communication is possible between higher level(Multiple Deme) single population at lower level Master Slave.

Thus study of various GAs are done and overall analysis is made. In the proposed work, implementation is to be made on Distributed Sensor Networks. Hence detailed search is done for selecting best suitable Genetic Algorithm from above explained methods. Consequently The Fined Grained GA having single population is found to be suitable.

\section{PROPOSED PLAN OF WORK}

Existing Work done is as follows:

The implemented work is all about Wireless Sensor Network WSN framed with GA. Each sensor node in WSN has limited wireless computational power to process 
and transfer the live data to the base station or data collection center. Generally, each sensor node has a low level of battery power that cannot be replenished. When the energy of a sensor node is exhausted, wireless sensor network leaks will appear, and the failed nodes will not relay data to the other nodes during transmission processing. The simulation started with the network setup phase, in which we have created a network of a predefined number of nodes; each node was assigned an $\mathrm{x}$ and $\mathrm{y}$ axis location and initialized with 2.0 Joules of energy. In a GA, Fitness was evaluated by the function defining the problem. The fitness of a chromosome was designed to minimize the energy consumption and to extend the network life time.

\section{Now moving towards current plan:}

Nodes Deployment: This is very initial step to be taken in case of Distributed Sensor Networks. In this particular algorithm the deployment of nodes in a particular area is done. Each node is assigned with node ID. This will position the nodes in the given area.

Routing Table allocation: The Routing tables are formed according to the deployment. The routing table will contain information about other nodes in the network in terms of node id and distance of each node with respect to other nodes in the network.

Route Evaluation: The route is to be calculated from the source node to destination node with the aid of using the control packets and the route must be found with respect to distance so that battery consumption is reduced and overall network remains healthy.

Node Failure Detection: By implementing Genetic Algorithm we can evaluate the faulty nodes. Those nodes whose battery power is less than standard value determined for a healthy network.

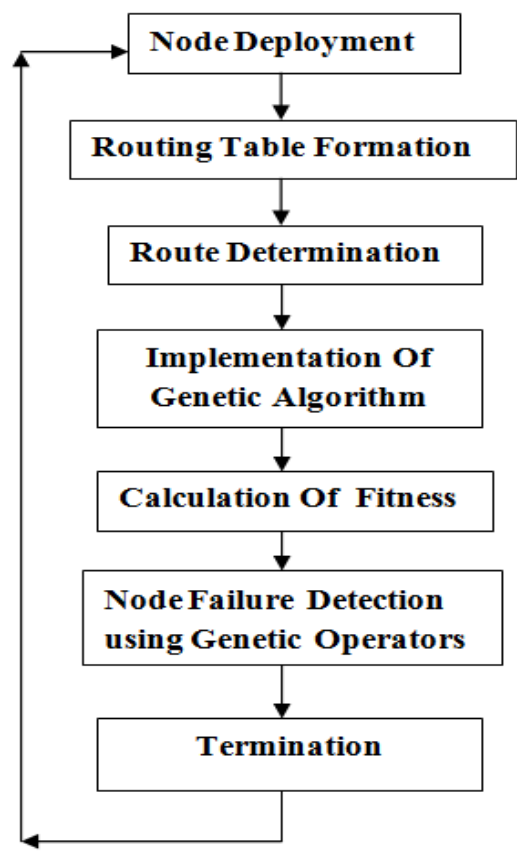

Figure: Flow_Chart of Overall work
Node Recovery: Once the faulty nodes are detected, they are removed in next generation of population while implementing Genetic Algorithm. Thus damaged nodes are replaced with the new nodes but with same node id. This can be more easily understood using following Flowchart.

\section{CONCLUSION}

Thus the population size for the network is generated. Selection two chromosomes with lowest fitness values based on Fitness Function is done. To perform fault detection of each node genetic operations such as: crossover and mutation operations are applied to two selected chromosomes. Thus construction of the active nodes in the DSN environment is established. Sink node takes the action upon receiving the information from sensor nodes. This above process is being implemented in proposed project. Finally the Fault Tolerance mechanism is the aim to be accomplished which is energy efficient and responsive to network in DSN.

Hence Very first step to be completed at primary stage is analysis and survey of various algorithms of different forms suitable. Once the foundation of work is done, the implementation is as efficient. Thus Paper presents the survey of classification of various types of Genetic Algorithms. In this way this survey helped to set up deployment task and proved to be important in the proposed work.

\section{REFERENCES}

[1] D. E. Goldberg, "Genetic Algorithms in Search, Optimization, and Machine Learning, Addison Wesley, Reading, MA, 1989.

[2] E-Book on "FUNDAMENTALS OF WIRELESS SENSOR NETWORKS THEORY AND PRACTICE" by Waltenegus Dargie Technical University of Dresden, Germany Christian Poellabauer University of Notre Dame, USA.

[3] Lokesh B. Bhajantri, N. Nalini, S. R. Nagaraj, H. V. Chaitra and N Srinivas "Performance Analysis of Heuristic Techniques for Optimization of Routing in Distributed Sensor Networks". 1Department of Information Science and Engineering, Basaveshwar Engineering College, Bagalkot, Karnataka, India.

[4] N. Thangadurai, Dr. R. Dhanasekaran, and R. Pradeep. "Energy Efficient Genetic Algorithm Model for Wireless Sensor Networks." International Journal of Computer Science and Electronics Engineering (IJCSEE) Volume 1, Issue 2 (2013) ISSN 2320-4028

[5] M. Senthil, K. Sugashini, M. Abirami, N. Vaigai . "Identification and Recovery of Repaired Nodes Based On Distributed Hash Table in WSN" Department of Computer Science and Engineering, Christ College of Engineering \& Technology,Puducherry, India.

[6] Ravneet Kaur, Neeraj Sharma. "Dynamic Node Recovery for Improved Throughput in MANET" Dept. of Computer Science and Engineering Chandigarh Engineering College,Mohali, India. Head of Dept. (Computer Science and Engineering) Chandigarh Engineering College ,Mohali, India.

[7] Implementation of Trust Aware Routing Framework With Link Failure Consideration and Recovery-Prof. Prashant P. Rewagad Head of the Department, Computer Science \& Engineering G. H. Raisoni College of Engineering and Management Jalgaon, Maharashtra, India.

[8] S.Sathish, A. Lawrance Ramesh, G. Sarath Kumar. A Survey on Node Recovery from a Failure in Wireless Sensor Networks. PG Scholars, Dept. of CSE Anna University Regional Centre, Coimbatore, Tamil Nadu, India respectively. 